\title{
The CO/HO system reverses inhibition of mitochondrial biogenesis and prevents murine doxorubicin cardiomyopathy
}

\author{
Hagir B. Suliman, ${ }^{1,2}$ Martha Sue Carraway, ${ }^{1}$ Abdelwahid S. Ali, ${ }^{2}$ \\ Chrystal M. Reynolds, ${ }^{1}$ Karen E. Welty-Wolf, ${ }^{1}$ and Claude A. Piantadosi ${ }^{1}$ \\ 1Department of Medicine and 2Department of Anesthesiology, Duke University Medical Center, Durham, North Carolina, USA.
}

\begin{abstract}
The clinical utility of anthracycline anticancer agents, especially doxorubicin, is limited by a progressive toxic cardiomyopathy linked to mitochondrial damage and cardiomyocyte apoptosis. Here we demonstrate that the post-doxorubicin mouse heart fails to upregulate the nuclear program for mitochondrial biogenesis and its associated intrinsic antiapoptosis proteins, leading to severe mitochondrial DNA (mtDNA) depletion, sarcomere destruction, apoptosis, necrosis, and excessive wall stress and fibrosis. Furthermore, we exploited recent evidence that mitochondrial biogenesis is regulated by the $\mathrm{CO} / \mathrm{heme}$ oxygenase $(\mathrm{CO} / \mathrm{HO})$ system to ameliorate doxorubicin cardiomyopathy in mice. We found that the myocardial pathology was averted by periodic $\mathrm{CO}$ inhalation, which restored mitochondrial biogenesis and circumvented intrinsic apoptosis through caspase-3 and apoptosis-inducing factor. Moreover, $\mathrm{CO}$ simultaneously reversed doxorubicin-induced loss of DNA binding by GATA-4 and restored critical sarcomeric proteins. In isolated rat cardiac cells, HO-1 enzyme overexpression prevented doxorubicin-induced mtDNA depletion and apoptosis via activation of Akt1/PKB and guanylate cyclase, while HO-1 gene silencing exacerbated doxorubicin-induced mtDNA depletion and apoptosis. Thus doxorubicin disrupts cardiac mitochondrial biogenesis, which promotes intrinsic apoptosis, while $\mathrm{CO} / \mathrm{HO}$ promotes mitochondrial biogenesis and opposes apoptosis, forestalling fibrosis and cardiomyopathy. These findings imply that the therapeutic index of anthracycline cancer chemotherapeutics can be improved by the protection of cardiac mitochondrial biogenesis.
\end{abstract}

\section{Introduction}

Doxorubicin (DOX) is frequently employed to treat hematological and solid tumors including leukemia, soft tissue sarcomas, and breast cancer. Despite its efficacy, the drug is associated therapeutically with a dilated cardiomyopathy (1), and more than a quarter of patients who receive DOX develop significant cardiac morbidity (2). DOX cardiotoxicity entails the generation of ROS by mitochondria - distinct on a molecular basis from its antineoplastic activity, which involves sequence-selective DNA intercalation and inhibition of topoisomerase II (3). Although ROS generation is a recognized pathogenic factor in DOX cardiomyopathy, low molecular weight antioxidants do not significantly reduce cardiac injury. However, ROS production is linked to cardiomyocyte apoptosis $(4,5)$, which is integral to the cardiomyopathy through the maladaptive structural changes that develop to increase physiological wall stress $(6,7)$.

Mitochondrial damage is thought to be central to the pathogenesis of DOX cardiomyopathy because the onset and severity of cardiomyocyte injury correlates with mitochondrial ROS production and disruption of bioenergetics $(8,9)$. Thus although mitochondria are important in DOX-induced apoptosis and necrosis, it is not understood why the mitochondriopathy is progressive or why the heart's response is so limited - especially since the myo-

Nonstandard abbreviations used: AIF, apoptosis-inducing factor; COX, cytochrome $c$ oxidase; DOX, doxorubicin; HO-1, heme oxygenase-1; MHC, myosin heavy chain; mtDNA, mitochondrial DNA; mtGFP, mitochondrial GFP; NRF-1, nuclear respiratory factor 1 ; OXPHOS, oxidative phosphorylation; sGC, soluble guanylate cyclase; SOD, superoxide dismutase.

Conflict of interest: The authors have declared that no conflict of interest exists. Citation for this article: J. Clin. Invest. 117:3730-3741 (2007). doi:10.1172/JCI32967. cardium is endowed with abundant mitochondrial antioxidant enzymes such as superoxide dismutase-2 (SOD2). Also, the heart normally has a robust mitochondrial turnover through autophagy (10) and mitochondrial biogenesis (11).

The cardiac antioxidant defenses also include heme oxygenase-1 (HO-1), an inducible enzyme that converts potentially toxic heme, e.g., that released by mitochondria, into the antioxidant biliverdin (12). Heme catabolism also releases iron and CO, which independently upregulate the cell's iron-responsive and antioxidant defenses. $\mathrm{CO}$ also has endogenous signaling properties, for instance, in the vasculature, where it relaxes smooth muscle by activating soluble guanylate cyclase (sGC), which is similar to but less potent than NO (13). Moreover, the eNOS-sGC system regulates mitochondrial biogenesis $(14,15)$, as does $\mathrm{CO}$, also in part via sGC, but independently of eNOS (16). CO further binds the reduced $a_{3}$ heme of cytochrome $c$ oxidase (COX), which enhances mitochondrial $\mathrm{H}_{2} \mathrm{O}_{2}$ release and contributes to retrograde activation of the nuclear mitochondrial biogenesis program via binding of the nuclear respiratory factor 1 (NRF-1) transcription factor with the PGC- $1 \alpha$ coactivator to target genes $(16,17)$. These genes include the mitochondrial DNA (mtDNA) transcription factor Tfam, which is essential for mtDNA replication in oxidative phosphorylation (OXPHOS), genome maintenance, and mitochondrial biogenesis (16). Thus physiological CO can promote and restore the critical capacity for energy production in cardiac cells.

The progressive mitochondrial damage and mitochondrial initiation of apoptosis in DOX-induced cardiomyopathy (18), along with the antiapoptotic mitochondrial profile that accompanies stimulation of the $\mathrm{CO} / \mathrm{HO}$ system (19), support the idea that $\mathrm{CO}$ would oppose intrinsic apoptosis after DOX administration. Therefore, 
A

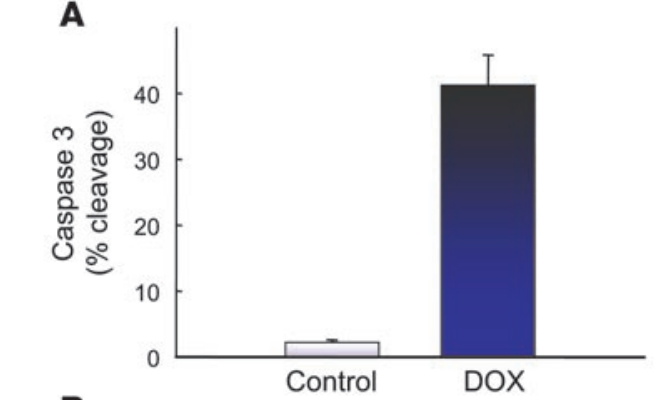

B
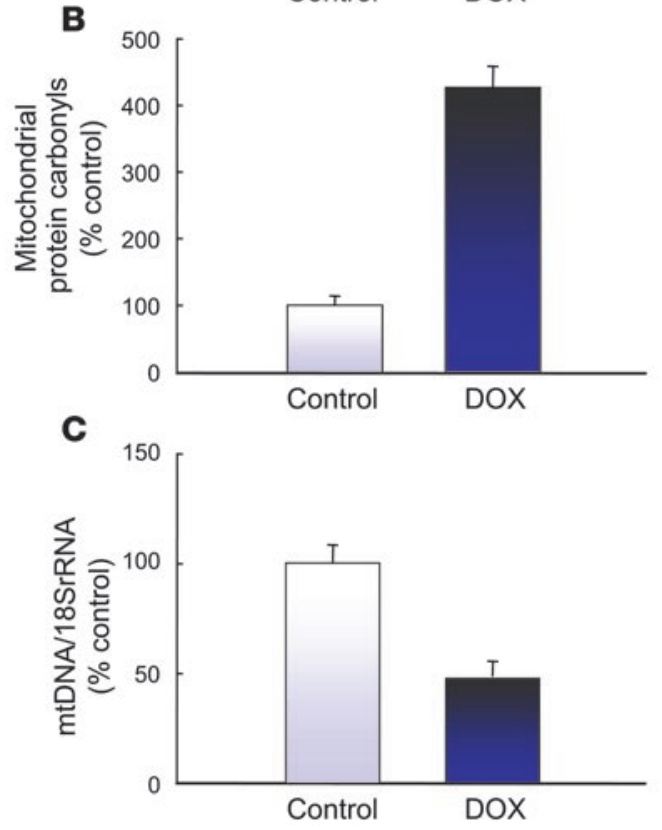

D

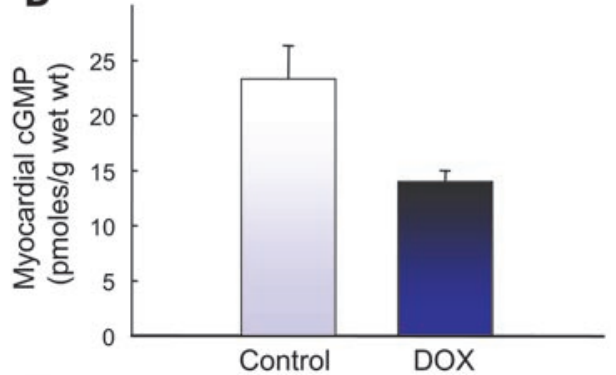

E

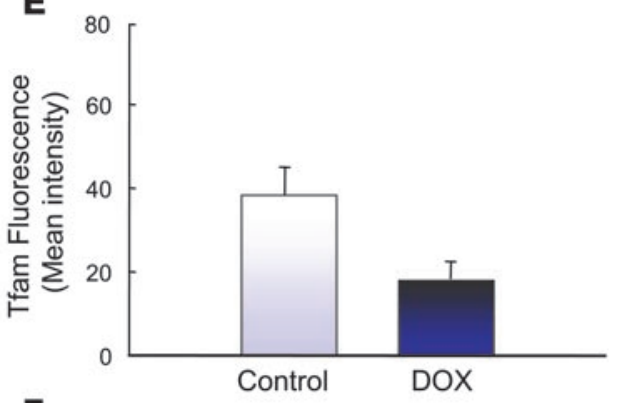

$\mathbf{F}$

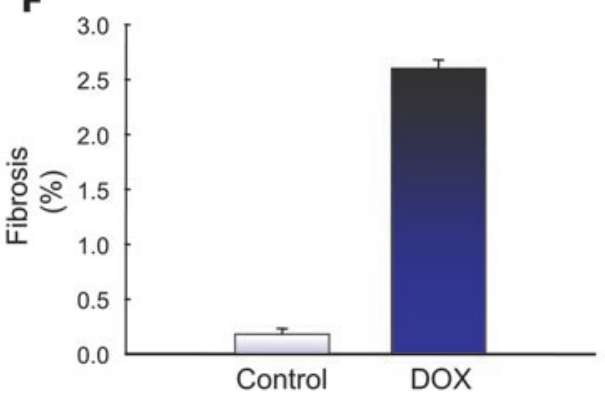

Figure 1

Cardiac injury in mice after DOX injection. The figure shows a 10 -fold increase in caspase-3 cleavage $(\mathbf{A} ; P<0.01)$, a 4 -fold increase in mitochondrial protein oxidation (B; $P<0.01$ ), a $50 \%$ reduction in mtDNA content $(\mathbf{C}$; $P<0.05$ ), and a $40 \%$ decrease in cardiac cGMP concentration (D; $P<0.05) 14$ days after a single DOX injection (15 mg/kg). In addition, the signal from the major transcription factor regulating mtDNA replication, Tfam, evaluated by in situ immunofluorescence, was reduced by $50 \%(E ; P<0.05)$. These changes were associated with a quantitative increase in cardiac fibrosis by Masson's trichrome staining $(\mathbf{F} ; P<0.01)$. using what we believe to be a novel combined molecular and morphological mitochondrial reporter approach (20), we tested the hypotheses that DOX inhibits cardiac mitochondrial biogenesis and that activation of mitochondrial biogenesis by periodic $\mathrm{CO}$ inhalation prevents the cardiomyopathy by protecting $\mathrm{mtDNA}$ and engendering an apoptosis-resistant mitochondrial phenotype.

\section{Results}

Molecular pathology. We quantified cardiac pathology in C57BL/6 mice after a single acute administration of DOX (15 mg/ $\mathrm{kg}$ i.p.) in which cardiomyocyte apoptosis and cardiac fibrosis culminate temporally at 14 days and remain stable for 28 days. Figure 1 shows the event profile at day 14 including a 10 -fold increase in caspase- 3 cleavage (Figure $1 \mathrm{~A} ; P<0.01$ ), a 4-fold increase in mitochondrial protein oxidation (Figure 1B; $P<0.01$ ), a 50\% reduction in mtDNA content (Figure 1C; $P<0.05$ ), and a $40 \%$ decrease in cardiac cGMP concentration (Figure 1D; $P<0.05$ ). In addition, mitochondrial Tfam, the transcription factor that regulates $m t D N A$ replication, was reduced by $50 \%$ by in situ immunofluorescence (Figure $1 \mathrm{E} ; P<0.05$ ). These changes led to an impressive increase in cardiac fibrosis quantified by Masson's trichrome staining (Figure 1F; $P<0.01$ ).

Cardiac wall stress and morphology. Mice given DOX developed cardiac degeneration and remodeling over the ensuing 3 to 14 days, and by day 14 the LV, measured at a constant intracavitary pressure, had become dilated and the $\mathrm{LV}$ wall thinner relative to control mice $(1.4 \pm 0.1 \mathrm{~mm}$ versus $2.1 \pm 0.1 \mathrm{~mm})$. Changes in DOX- induced cardiac wall stress and morphology were evaluated without and with CO inhalation on 2 regimens: 1 hour of 500 ppm CO 24 hours before DOX, or 24 hours before and again 7 days after DOX administration. Control mice were exposed to an analogous regimen of hypoxic hypoxia $(\mathrm{HH})$ without or with DOX in order to assess the role of hypoxia in the $\mathrm{CO}$ responses.

As shown in Figure 2A, DOX mice receiving 1 CO treatment developed less cardiac wall stress compared with controls, and mice given 2 treatments showed normalization of LV chamber cross-sectional area and wall thickness $(1.6 \pm 0.1 \mathrm{~mm}$ after $1 \mathrm{CO}$ treatment, and $1.9 \pm 0.1 \mathrm{~mm}$ after $2 \mathrm{CO}$ treatments). Control mice receiving $\mathrm{CO}$ with no DOX showed minor increases in $\mathrm{LV}$ wall thickness $(2.3 \pm 0.1 \mathrm{~mm})$. In contrast, the hearts of mice given 1 (data not shown) or 2 treatments of $\mathrm{HH}$ (Figure 2A) were not protected significantly. These data indicate that $\mathrm{CO}$ mitigates the DOX-mediated derangement in LV contractile geometry through direct effects, not by hypoxia.

Sections of mouse heart stained with H\&E were examined by light microscopy (Figure 2A). Heart sections from control and CO-treated mice were indistinguishable by histology and appeared normal morphologically. The DOX heart was characterized by loss of myofibrils and extensive cytoplasmic vacuolization, a hallmark of necrosis. DOX-treated mice given CO showed improved myocardial morphology, although after the single CO treatment, the myocytes still often showed vacuolization. HH had no effect on the cardiac histology, and mice given one (data not shown) or 2 treatments of $\mathrm{HH}$ were not protected. 
A

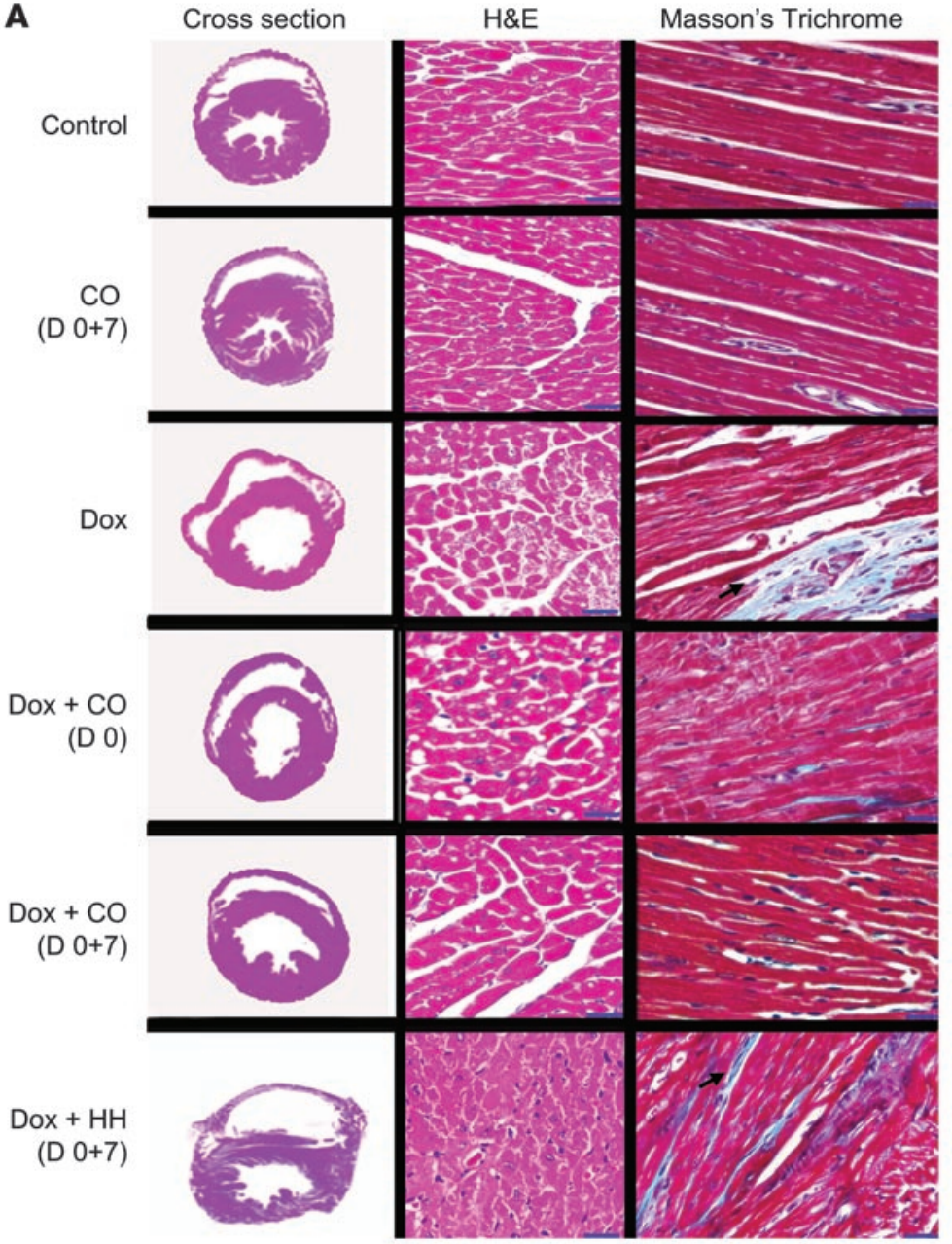

B

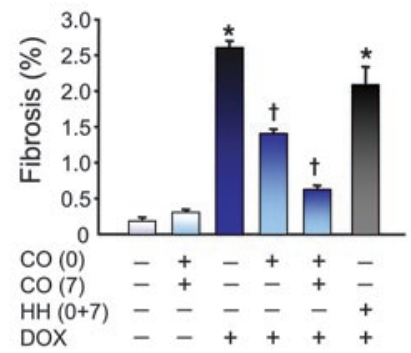

\section{Figure 2}

Pathology of mouse heart after DOX treatment. (A) Representative gross and microscopic pathology. Left column: Cross-sections of mouse hearts fixed at a constant intracavitary pressure illustrate DOX-induced cardiomyopathy after 14 days. Normal mouse heart (control), CO control (CO), and DOX cross-sections are shown. CO administration, but not $\mathrm{HH}$, preserves myocardial mass and LV wall thickness in DOX-treated mice. Middle column: Representative photomicrographs of LV sections of control, CO, DOX, and CO+DOX-treated mice stained with H\&E (original magnification, $\times 250$; scale bar $=10 \mu \mathrm{m}$ ). Normal myocardial morphology is shown (control). CO did not alter the myocardial morphology. DOX-damaged hearts showed extensive cytoplasmic vacuolization, myofibrillar loss, and cell death. Cardiomyocyte vacuolization and cell death were greatly reduced in animals that received $\mathrm{CO}$, but swelling and vacuolization were still prominent after $\mathrm{HH}$. Right column: Representative photomicrographs of mouse LV sections stained with Masson's trichrome. Arrows indicate fibrosis of endomysium (light blue staining). Mice treated with CO showed less fibrosis after DOX. (B) Quantification of the interstitial fibrosis. Each bar represents mean \pm SEM of 6 hearts. ${ }^{\star} P<0.01$ versus control; ${ }^{\dagger} P<0.05$ versus DOX. Note that $\mathrm{CO}$ protects, but $\mathrm{HH}$ does not.

DOX-treated mice also developed myocardial fibrosis after 14 days, as indicated by Masson's trichrome staining, which was avoided by $2 \mathrm{CO}$ exposures but not $2 \mathrm{HH}$ exposures. Image quantification indicated that DOX increased the area of fibrosis in the ventricular endomysium compared with control mice (control, $2.7 \pm 0.10 \%$, versus DOX, $0.42 \% \pm 0.05 \%$ ). In mice receiving $1 \mathrm{CO}$ dose, areas of DOX-induced fibrosis could still be found occasionally, while fibrosis was almost absent in mice treated with $2 \mathrm{CO}$ doses (Figure 2A; $0.74 \% \pm 0.07 \%$ ). Figure $2 \mathrm{~B}$ shows a quantitative assessment of cardiac collagen density (ratio of collagen area/total area), which confirms that $\mathrm{CO}$ but not $\mathrm{HH}$ attenuated myocardial fibrosis.

Cardioprotection from DOX-induced apoptosis. Intrinsic apoptosis, apart from necrosis, is central to DOX-induced cell death, and caspase-3 is the pivotal effector protein. In the DOX-treated mouse heart at
14 days, myocardial caspase- 3 cleavage had increased prominently, consistent with ongoing intrinsic apoptosis (Figure 3A). CO administration to DOX mice, however, greatly abrogated caspase- 3 activation. To confirm that $\mathrm{CO}$ blocked intrinsic apoptosis, we evaluated nuclear translocation of proapoptotic apoptosis-inducing factor (AIF) protein in mitochondrial and nuclear cell fractions by western blot analysis. The disruption of mitochondria releases AIF, which translocates to the nucleus and participates in DNA fragmentation (21). The ratio of nuclear-to-mitochondrial AIF in the heart increased more than 4-fold after DOX, an effect essentially eliminated by $2 \mathrm{CO}$ treatments (Figure 3A). Nuclear AIF in DOX-treated mice correlated with the observed increase in caspase- 3 cleavage $(r=0.88 ; P<0.01)$. Also, Figure $3 \mathrm{~A}$ (right 3 lanes) shows controls indicating that neither 1 nor 2 treatments of $\mathrm{HH}$ prevents caspase- 3 cleavage or AIF release. 
A
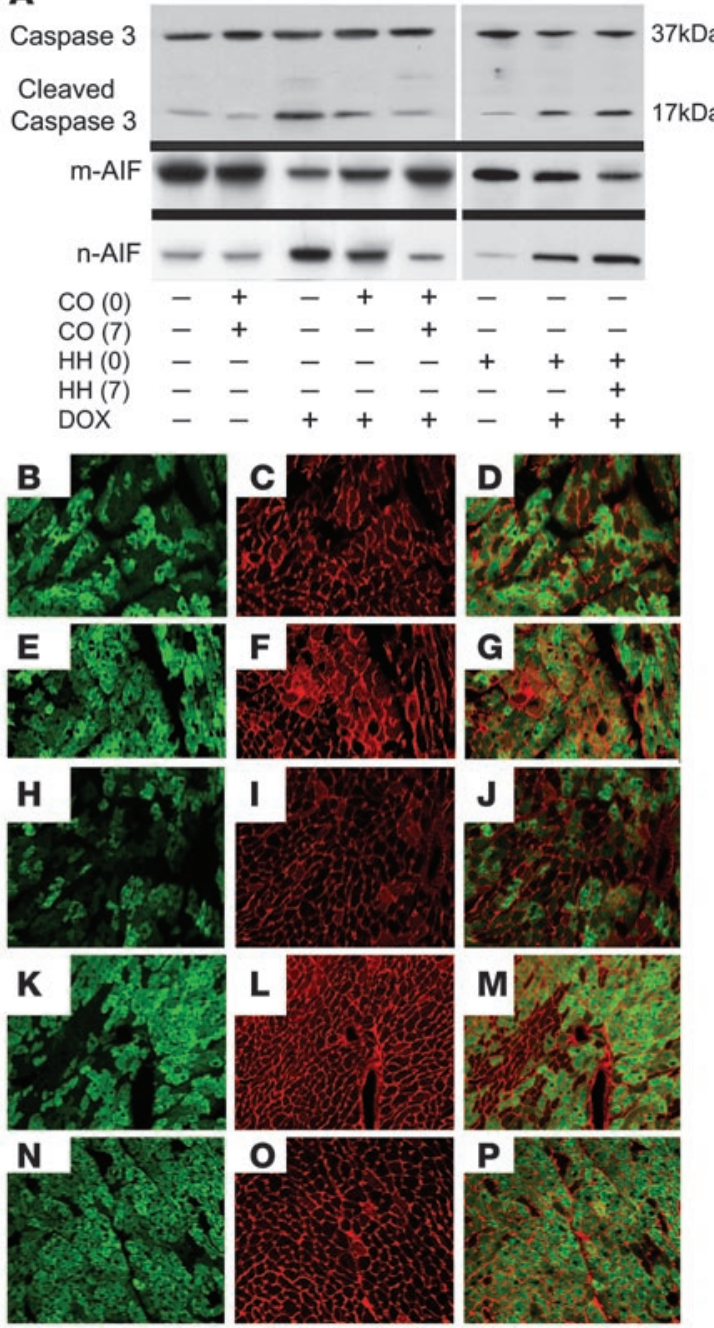

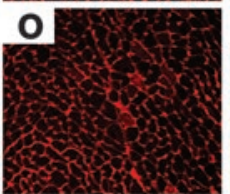

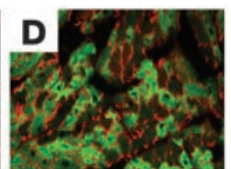
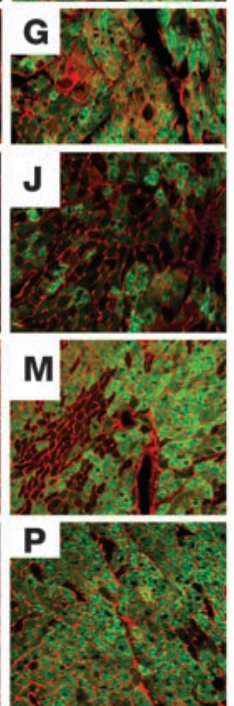
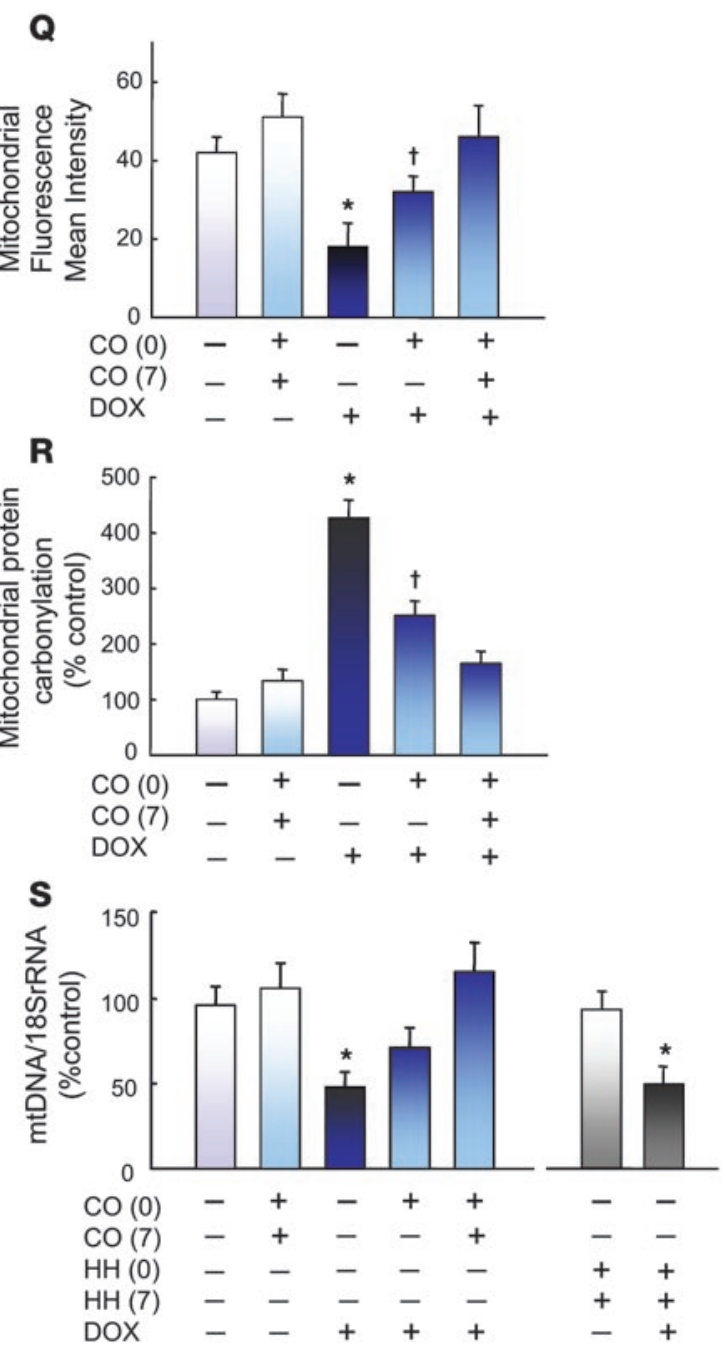

Figure 3

Apoptosis and mitochondrial damage after DOX treatment. (A) Western blot analysis of caspase-3 using antibody that recognizes the full-length molecule (37-kDa band) and the 17-kDa proteolytic-cleavage fragment. Western blot analysis was also performed using anti-AIF antibody that detects mitochondrial and nuclear AIF. Note that CO prevented caspase-3 cleavage and AIF release after DOX, but HH did not. (B-P) Photomicrographs of immunolabeling for laminin (red), GFP-mitochondria (green), and overlay in mouse heart. (B-D) Control LV shows intense laminin labeling in the endomysium framing the cardiomyocytes and small vessels. $\mathrm{CO}$ alone did not alter the distribution or intensity of laminin or mitochondrial fluorescence $(\mathbf{E}-\mathbf{G})$, whereas laminin in DOX-treated mice outlined both living and degenerating or dead cardiomyocytes by loss of intensity of mitochondrial fluorescence $(\mathbf{H}-\mathbf{J})$. Improvement in DOX-treated mice was seen after $1(\mathbf{K}-\mathbf{M})$ or 2 CO doses $(\mathbf{N}-\mathbf{P})$. Original magnification, $\times 300$. (Q) Quantification of mitochondrial fluorescence intensity relative to laminin. Values are mean \pm SEM data for 6 mice per group. (R) The effects of DOX and CO treatment on the protein carbonyl content of cardiac mitochondria by anti-dinitrophenol dot blotting and quantification by densitometry. Each bar graph represents mean \pm SEM of 6 mice. (S) Histogram of real-time PCR data for cellular mtDNA content showing decreases after DOX are prevented by CO but not by $\mathrm{HH}$. ${ }^{*} P<0.05$ versus other groups; ${ }^{\dagger} P<0.05$ versus control.

Transgenic GFP mitochondrial reporter mice. In order to investigate DOX-related changes in mitochondrial structure and the effects that $\mathrm{CO}$ had on it, the cardiac mitochondrial distribution was evaluated by fluorescence microscopy using reporter mice that express GFP constitutively in mitochondria (mtGFP-tg mice). This mouse allows direct visualization of mitochondrial density and distribution with GFP against other fluorescence markers, e.g., ones that delineate cell structure, in this case laminin (Figure 3, B-D). In the normal reporter mouse heart, laminin (red) staining was most intense in the endomysium where it outlines the myocytes, invaginates into the T-tubules, and is present around small vessels, espe- cially capillaries (Figure 3C). CO here produced little or no change in this staining pattern (Figure 3, E-G), whereas heart sections from mtGFP-tg mice receiving DOX demonstrated dramatic losses in mitochondrial staining relative to laminin (Figure 3, H-J). After DOX, the mean transverse cardiomyocyte diameter was significantly smaller than in control mice, a characteristic of atrophy $(12.7 \pm 0.1$ versus $14.6 \pm 0.1 \mu \mathrm{m}$ ). CO pretreatment partly prevented (Figure 3, $\mathrm{K}-\mathrm{M}$ ), and $2 \mathrm{CO}$ doses fully prevented (mean transverse diameter, $14.4 \pm 0.1 \mu \mathrm{m}$ ) the DOX-induced atrophy (Figure 3, N-P).

We examined the mean intensity of the mitochondrial fluorescence in randomly chosen cardiac sections of mtGFP-tg mice 
A

\begin{tabular}{|c|c|c|c|c|c|}
\hline COXI & $-=$ & $=$ & -- & -- & - \\
\hline $18 S$ & ت & كت & تص & ت & ت \\
\hline $\mathrm{CO}(0)$ & 一 & + & - & + & + \\
\hline $\mathrm{CO}(7)$ & - & + & - & - & + \\
\hline DOX & - & - & + & + & + \\
\hline
\end{tabular}

B

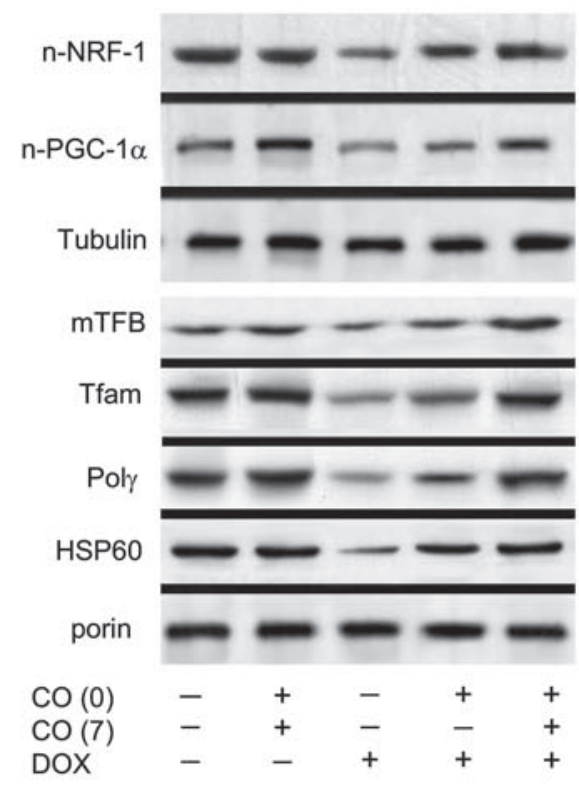

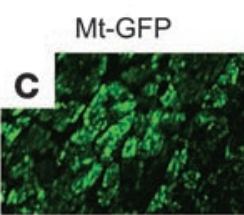
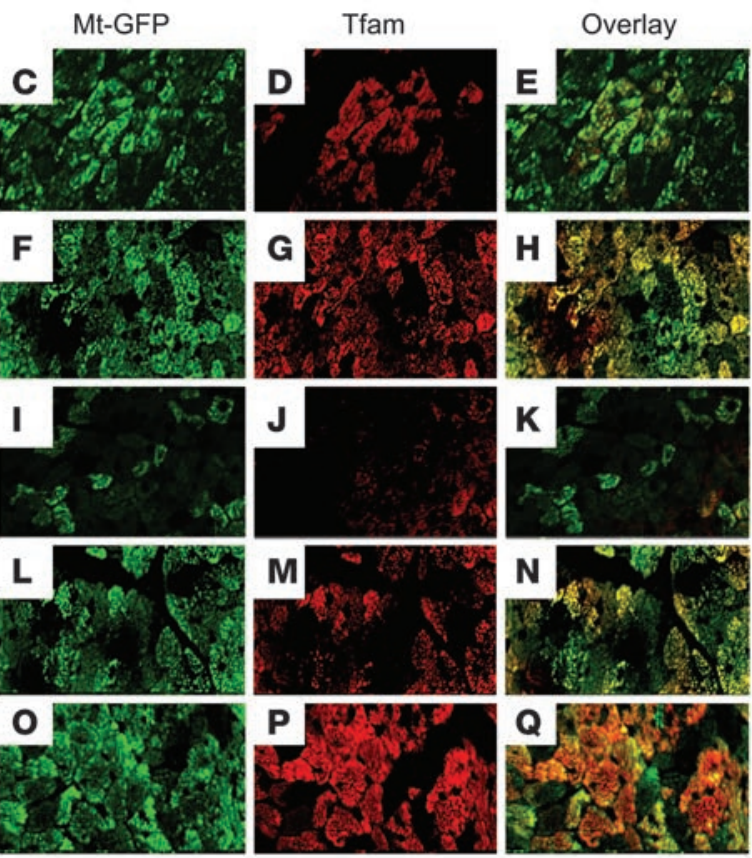

$\mathbf{R}$

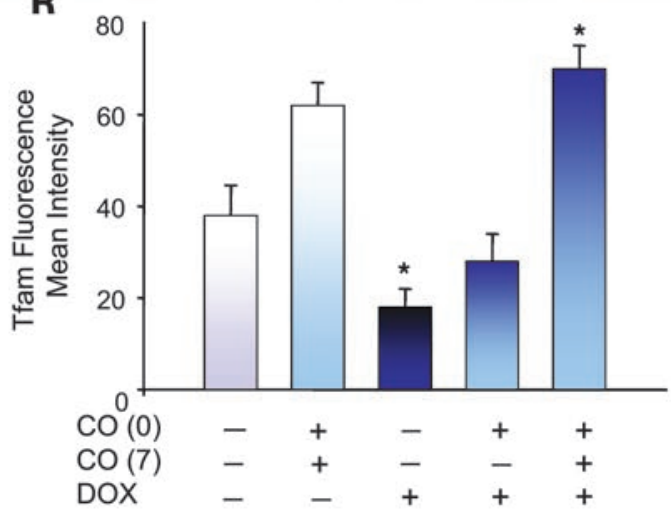

Figure 4

DOX inhibits and CO restores regulation of cardiac mtDNA replication and mitochondrial biogenesis. (A) Mitochondrial gene expression evaluated by RT-PCR using mouse gene-specific primers for mitochondrial COXI. Nuclear mRNA for $18 \mathrm{~S}$ rRNA was used to control for RNA integrity and RT-PCR efficiency. (B) Top: Expression of nuclear protein regulators of mitochondrial biogenesis, NRF-1 and PGC-1 $\alpha$, by western blot compared with tubulin. Bottom: Mitochondrial content of regulatory proteins for mtDNA replication by western blot. Mitochondrial mTFB, Tfam, Poly and HSP-60 are shown compared with porin. DOX effects are reversed by CO. (C-Q) Confocal scanning microscopy of immunolabeling for Tfam (red), mitochondria (green), and overlay in heart tissue. Control sections of LV showed intense Tfam labeling in mitochondria (C-E), and $\mathrm{CO}$ did not alter mitochondrial distribution $(\mathbf{F}-\mathbf{H})$. (I-K) LV sections of DOX-treated mice. Tfam was clearly labeled in mitochondria of intact but not degenerating or necrotic cardiomyocytes. Also shown are LV sections of mice treated with DOX plus 1 dose of CO (L-N) or DOX plus 2 doses of $\mathrm{CO}(\mathbf{O}-\mathbf{Q})$. Original magnification, $\times 300$. (R) Histogram of the Tfam fluorescence intensity relative to mitochondrial fluorescence. Values are mean \pm SEM of results of 6 mice per group. ${ }^{*} P<0.05$ versus other groups.

(Figure $3 \mathrm{Q}$ ) and found it was attenuated quantitatively by 14 days in mice receiving DOX compared with control mice. Mitochondrial fluorescence intensity was enhanced by CO treatment, and the 2-dose protocol prevented the loss of fluorescence after DOX. The distribution of GFP also substantiated mitochondrial proliferation morphologically after $\mathrm{CO}$ treatment, as multiple new fluorescent aggregates that appeared within the cardiomyocytes were confirmed by EM as having mitochondrial structure (16).

$C O$ prevents oxidative protein and $m t D N A$ damage. A prevalent indicator of oxidative protein damage in the heart is protein carbonyl accumulation (22), and cardiac mitochondria displayed a 5-fold increase in protein carbonylation in DOX mice after 14 days (Figure 3R). Carbonyl accumulation was decreased by $1 \mathrm{CO}$ pretreatment and almost eliminated by $2 \mathrm{CO}$ treatments. The second regimen had no significant effect on mitochondrial protein carbonyl levels in the hearts of naive control mice.

After DOX administration, ongoing damage to cardiac mtDNA and protein was still present at 14 days (Figure $3 \mathrm{~S}$ ). In the LV, the mtDNA copy number after DOX fell to half that of the control heart. In mice receiving $1 \mathrm{CO}$ dose, mtDNA depletion by DOX was lessened by half, to $74 \%$ of control (Figure 3S). In mice receiving 2 CO treatments, cardiac mtDNA copy number after DOX did not 
A

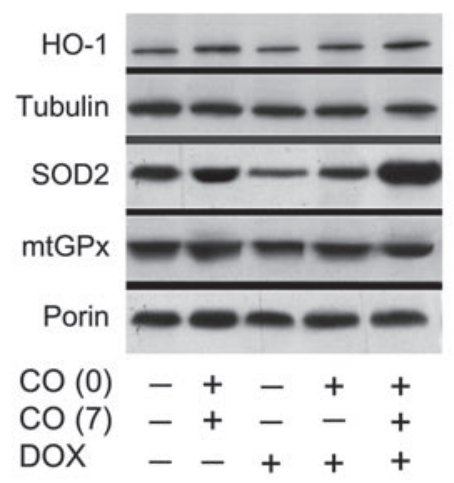

$\mathbf{Q}$

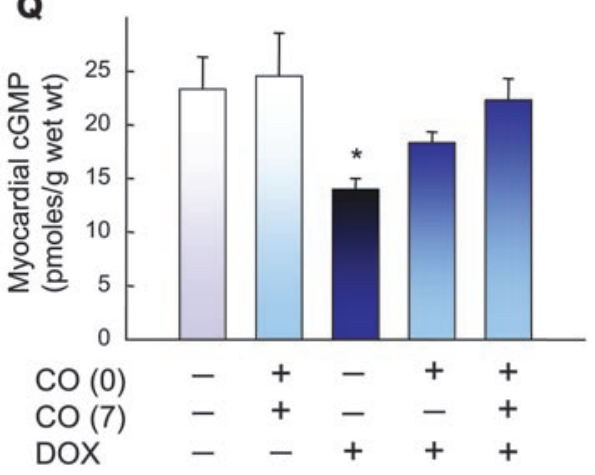

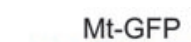
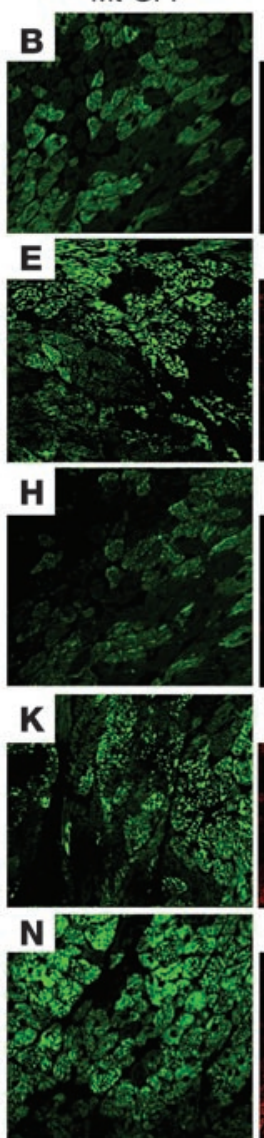
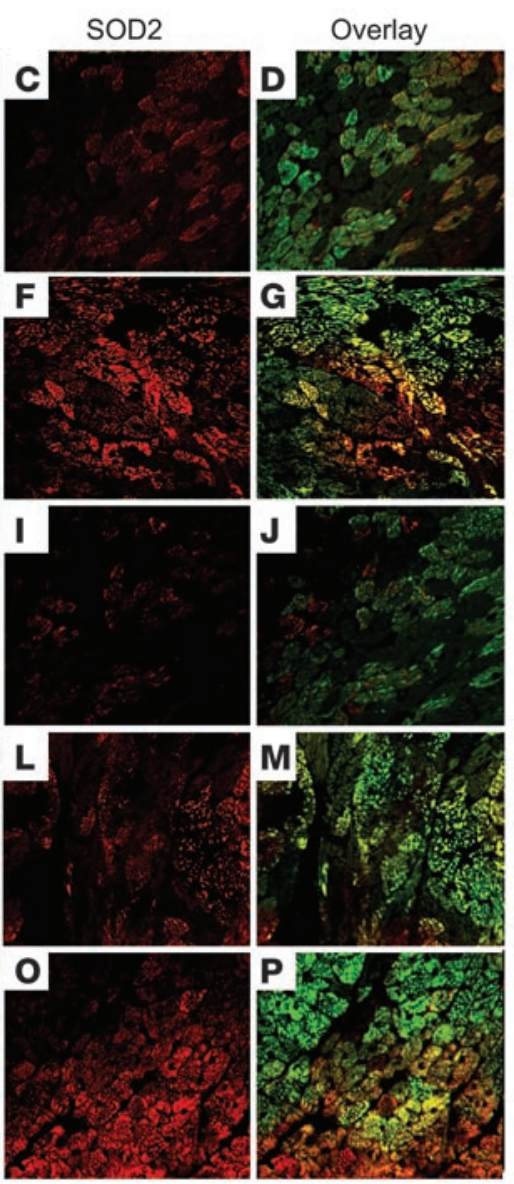

\section{Figure 5}

CO restores mitochondrial antioxidant enzyme and cardiac cGMP content in DOX-treated mice. (A) Western blots of cardiac HO-1 (compared with tubulin) and mitochondrial SOD and glutathione peroxidase (mtGPX) relative to porin. (B-P) Fluorescence microscopy localizing SOD2 (red) to the mitochondria (green). Control LV showed intense SOD2 labeling in cardiomyocyte mitochondria (B-D); CO increased mitochondrial staining intensity $(\mathbf{E}-\mathbf{G})$; sections of LV in DOX-treated mice after 14 days $(\mathbf{H}-\mathbf{J})$ showed scattered SOD2 labeling in mitochondria; LV of mice treated with DOX plus 1 dose of $\mathrm{CO}(\mathbf{K}-\mathbf{M})$ and $L V$ of mice treated with DOX plus 2 doses of $C O(\mathbf{N}-\mathbf{P})$ were improved. The histogram $(\mathbf{Q})$ demonstrates loss of cGMP in myocardium after DOX was restored by CO. Values are means \pm SEM of 6 experiments. ${ }^{*} P<0.05$ versus other groups. Original magnification, $\times 300$.

fall below control values. Neither 1 treatment (data not shown) nor 2 treatments with $\mathrm{HH}$ (Figure 3S) prevented the DOX-induced loss of mtDNA copy number.

Cardiac OXPHOS gene expression and mitochondrial biogenesis. In order to relate the mitochondrial pathology to the protective molecular function of $\mathrm{CO}$, we measured mitochondrial mRNA transcript levels for the first integral mtDNA-encoded gene compared with a nuclear housekeeping gene (Figure 4). Figure 4A shows that steady-state mitochondrial COX subunit I (COXI) mRNA levels are reduced after DOX relative to $18 \mathrm{~S}$ rRNA. This decrease in COXI gene transcription is attenuated by CO pretreatment and abrogated by 2 treatments. Thus the decline in mtDNA copy number caused by DOX significantly depresses mtDNAencoded gene expression, and CO opposes this effect.

The ability to overcome mitochondrial damage entails activation of mitochondrial biogenesis; however, in tracking 2 nuclear proteins integral to the transcriptional control of mitochondrial gene expression, NRF-1 and the coactivator PGC-1 $\alpha$, we found that DOX severely depresses mitochondrial biogenesis in the heart (Figure 4B). DOX also decreased the protein levels for mitochondrial transcription factors A and B (Tfam, mTFB) - imported regulators of mtDNA transcription and replication - as well as Pol $\gamma$, the DNA polymerase required for mtDNA transcription and replication. CO counteracted these effects, and the level of these mitochondrial proteins improved in DOX mice after 2 CO doses (Figure 4B). DOX-treated mice also showed significant depression of mitochondrial HSP-60 protein expression (by 51\%), and this loss of HSP-60 was prevented by CO (Figure 4B).

The functional failure of the mitochondrial transcriptosome after DOX-induced mitochondrial damage was corroborated using Tfam immunofluorescence. Tfam protein in the mitochondria tracks myocardial mitochondrial biogenesis, and in this study it correlated roughly with the $\mathrm{mtDNA} / \mathrm{nDNA}$ ratio $\left(P=0.004, r^{2}=0.48\right)$ and COXI expression $\left(P=0.005, r^{2}=0.48\right)$. DOX thus interfered not only with expression of nuclear-encoded mitochondrial transcriptosome and chaperone proteins, but inevitably, a failure to import mitochondrial Tfam and HSP-60 led to mtDNA depletion and loss of OXPHOS capacity. In mtGFP-tg reporter mice, cardiac 


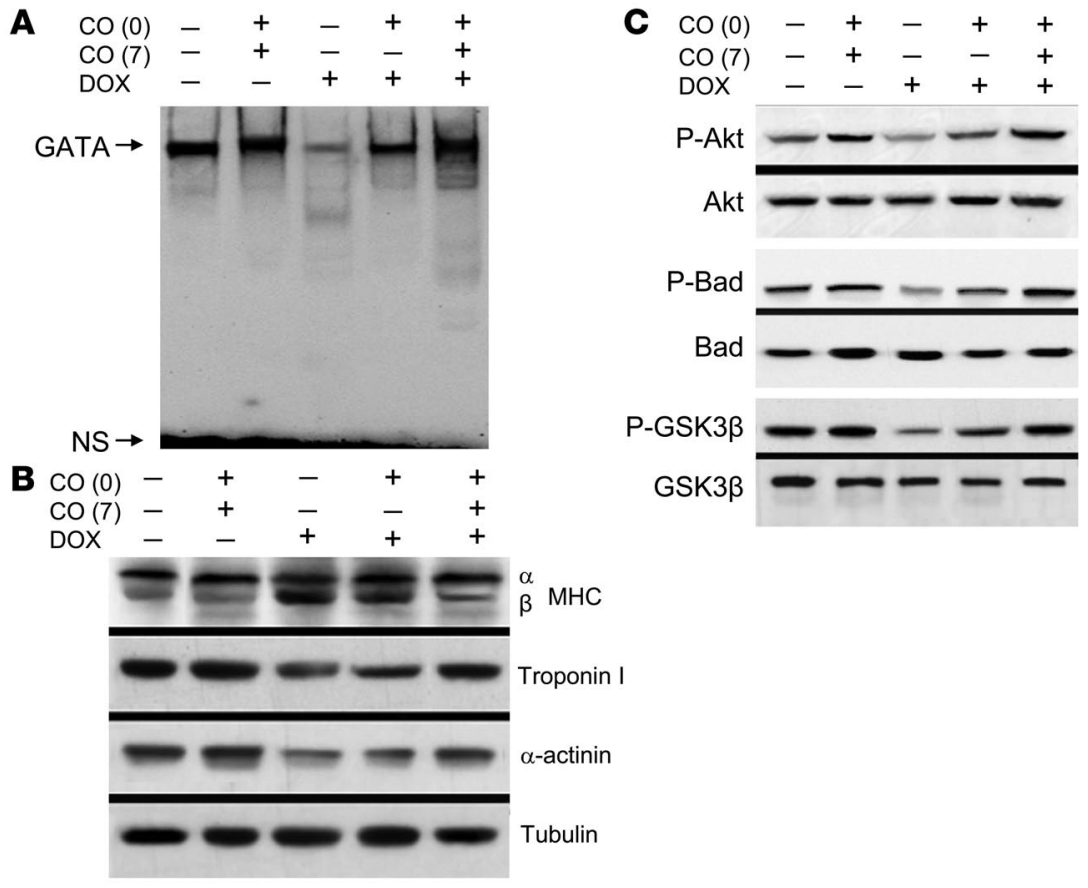

\begin{abstract}
Figure 6
Apoptosis and cardiomyocyte differentiation after DOX. (A) CO abrogated the loss of GATA-4 DNA binding activity after DOX. PAGE showing GATA-4 DNA binding activity of nuclear protein $(10 \mu \mathrm{g})$ by EMSA. The top arrow denotes the specific proteinDNA complex. The bottom arrow shows the nonspecific (NS) free probe. A 100-fold molar excess of cold consensus or mutant oligonucleotides confirmed the binding specificity in competition studies (data not shown). (B) Myosin heavy chain (MHC) isoforms in LV homogenates after silver staining. Western blot shows the DOX effect on myocardial troponin I and $\alpha$-actinin relative to tubulin expression. (C) Top: Western blot of pAkt/Akt ratio in mouse heart before and after $\mathrm{CO}$ treatment and with or without DOX. Middle: Western blot of total and phospho-Bad demonstrates enhanced phosphorylation of the antiapoptotic protein in the heart after CO. Bottom: Cardiac GSK3 $\beta$ protein by western analysis shows an increase in pGSK3 $\beta / G S K 3 \beta$ ratio after $\mathrm{CO}$.
\end{abstract}

mitochondria colocalized with Tfam immunoreactivity, as shown in Figure 4, C-E, which was not altered by CO (Figure 4, F-H). Nuclear cardiomyocyte immunofluorescence was not observed, and the mitochondrial Tfam signal was blocked by preincubation with the original peptide prior to staining (data not shown). In DOX-treated reporter mice, mtGFP declined as expected from the cell loss (Figure 4I) as well as from a slight fall-off in GFP expression (not shown). Tfam staining is greatly reduced (Figure 4, $\mathrm{J}$ and $\mathrm{K}$ ); however, this is restored by $2 \mathrm{CO}$ treatments (Figure 4, $\mathrm{O}$ and $\mathrm{P}$ ). These observations were checked by quantitative immunofluorescence, which indicated that DOX mice given $2 \mathrm{CO}$ doses had twice as much cardiac Tfam staining as mice given DOX alone (Figure 4R). Collectively, Figure 4 confirms that $\mathrm{CO}$ regulates mitochondrial biogenesis by increasing the nuclear expression of NRF-1, PGC-1 $\alpha$, and Tfam. These responses are necessary to maintain mitochondrial biogenesis, which helps preserve cardiac mtDNA content in the DOX-exposed mouse heart.

Antioxidant defenses and cGMP in the mouse heart. The responses to DOX-induced oxidative stress and the influence of $\mathrm{CO}$ were monitored via the expression of 3 antioxidant enzymes with antiapoptotic effects, HO-1, mitochondrial SOD (SOD2), and mitochondrial GPX (mGPX). In DOX-treated mice, the expression of these enzymes in the heart by western blot analysis, especially SOD2 expression, was reduced at 14 days (Figure 5A). Without DOX, the 2-dose $\mathrm{CO}$ regimen increased $\mathrm{HO}-1$ and SOD2 but not mGPX expression, while after DOX, these proteins were maintained by CO treatment at or above the levels found in naive control mice.

To localize cardiac SOD2 expression, mtGFP-tg reporter mice were used in combination with immunofluorescence, and a relatively uniform distribution of enzyme was found that localized strongly to the myocardial mitochondria (Figure 5, B-P). In agreement with the western blots, the DOX mouse heart showed many fewer SOD2-positive cardiomyocytes, while mice treated with $\mathrm{CO}$ alone or with DOX and CO maintained a normal distribution of SOD2 (Figure 5, B-P).
Mitochondrial biogenesis is regulated in part by sGC, which can be activated by $\mathrm{NO}$ or $\mathrm{CO}$, and cyclic GMP signaling helps maintain mitochondrial content. The activation of sGC by CO therefore would oppose mitochondrial damage by DOX because the drug destabilizes NO signaling (23). We first confirmed that DOX depresses mean myocardial cGMP levels from $23.8 \pm 0.2 \mathrm{pmol} / \mathrm{g}$ protein in controls to $14 \pm 1.0 \mathrm{pmol} / \mathrm{g} 14$ days after DOX treatment (Figure 5Q) and then showed that this reduction in cardiac cGMP is prevented by the 2 -dose CO regimen $(24.2 \pm 0.2 \mathrm{pmol} / \mathrm{g})$.

GATA activation and sarcomeric proteins expression. Cardiac sarcomere protein expression is regulated in part by GATA family transcription factors $(24,25)$, and DOX interferes with GATA-DNA binding activity $(26,27)$. GATA binding activation was analyzed by EMSA in cardiomyocyte nucleoprotein, and gel supershifts showed the active protein in mouse heart to be mostly GATA-4. Significantly lower GATA-4 DNA binding activity was demonstrated in DOX-exposed hearts compared with controls (Figure 6A), and inhibition of GATA-DNA binding activity by DOX is reversed after CO (Figure 6A). DNA binding competition studies with cold oligonucleotide confirmed the specificity, and GATA-4 binding was not observed when ${ }^{32} \mathrm{P}$-labeled oligonucleotide with a mutated sequence was used (TCTTA instead of TGATAA; negative control data not shown).

An important hallmark of cardiomyocyte degeneration is loss of sarcolemmal integrity. In the mouse heart, DOX affects the major sarcomere proteins - troponin I, $\alpha$-actinin, and both major myosin heavy chain (MHC) isoforms. The distribution of MHC isoforms shown in Figure 6B indicates that the slow $\beta$-MHC isoform increases in DOX mice without a significant change in the $\alpha$-MHC isoform. DOX also significantly decreases cardiac troponin I levels relative to tubulin, reflecting a breakdown in membrane integrity, and loss of $\alpha$-actinin, a component of the $\mathrm{Z}$-line, reflects disruption of myofibrils (Figure 6B). The 2-dose $\mathrm{CO}$ regimen prevents loss of troponin I and $\alpha$-actinin $(P<0.05$ by densitometry analysis). 
A

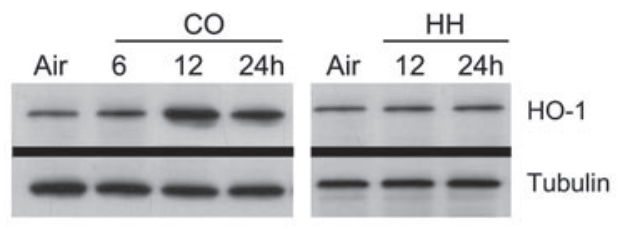

B

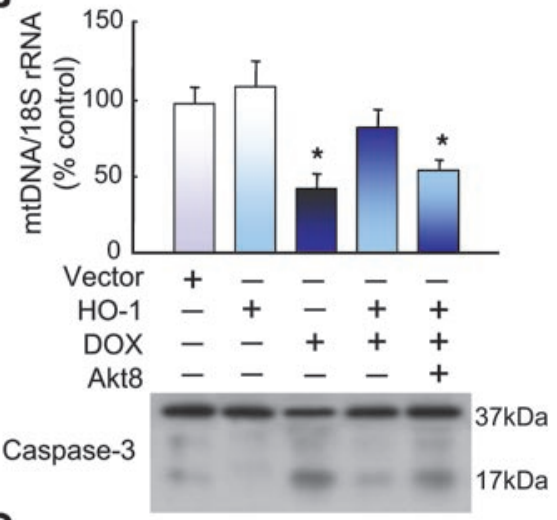

C

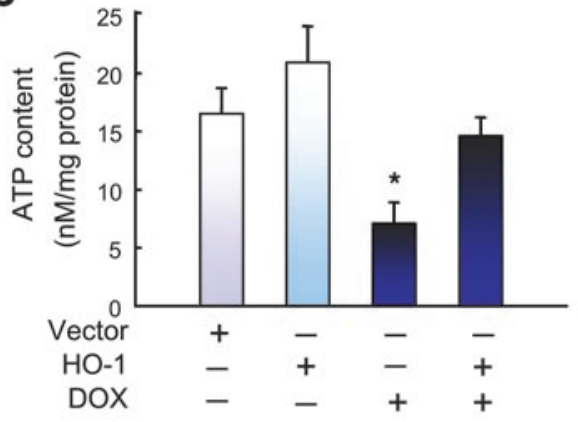

D

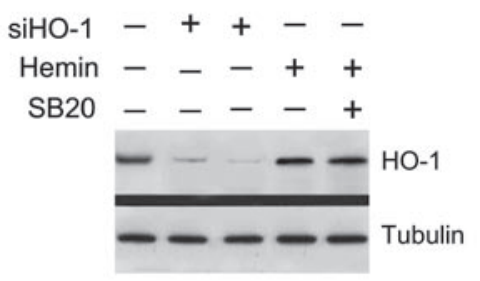

E

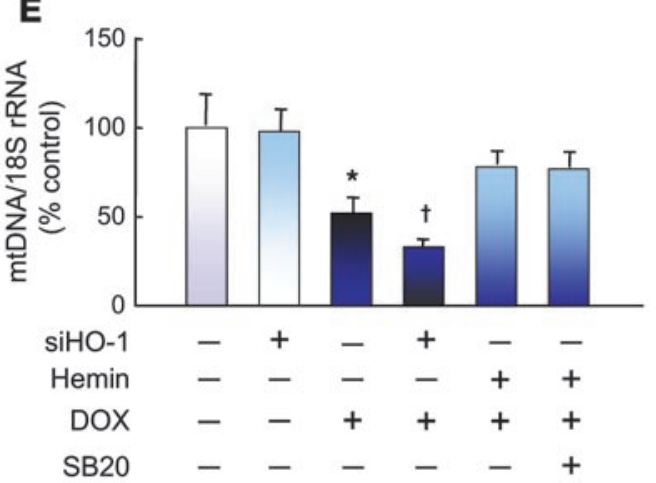

\section{Figure 7}

HO-1 protects against DOX-induced mtDNA damage and apoptosis. (A) Western blots of HO-1 expression before and after CO or HH in control mouse hearts compared with tubulin. (B) Bar graph shows mtDNA copy number measured by real-time PCR relative to nuclear DNA in H9c2 cells before and after HO-1 gene transfer. HO-1 transfection decreased DOX-dependent mtDNA depletion. HO-1-transfected cells treated with the specific Akt inhibitor (Akt8) showed loss of mtDNA protection by HO-1. HO-1 gene transfer also protected H9c2 cells from DOX-induced apoptosis, as shown by western blot analysis with an antibody to both intact and cleaved caspase-3. In HO-1-transfected H9c2 cells preincubated with Akt8, caspase-3 cleavage reemerged (mean \pm SEM, $n=3$; ${ }^{*} P<0.05$ versus untreated cells). (C) Effect of DOX on cellular ATP content and the rescue by HO-1 transfection. ${ }^{\star} P<0.05$ compared with control and both $\mathrm{HO}-1$ transfection studies. (D) $\mathrm{HO}-1$ silencing in $\mathrm{H} 9 \mathrm{c} 2$ cells. Western blot analysis demonstrated loss of $\mathrm{HO}-1$ expression at 24 and 48 hours after $\mathrm{HO}-1$ siRNA. Control cells stimulated with hemin ( $50 \mu \mathrm{M})$ were the positive control. Cells pretreated with p38 MAPK inhibitor SB203580 (10 $\mu \mathrm{M})$ did not show a change in HO-1 protein induction by hemin after an additional 6 hours. (E) mtDNA copy number measured by real-time PCR in H9c2 cells without or with HO-1 silencing. Control or siHO-1 transfected cells were preincubated for 24 hours with DOX alone, with DOX and hemin, or with DOX, hemin, and SB203580. The graphs are means \pm SEM for $n=3$ per group. ${ }^{*} P<0.05$ compared with control; ${ }^{\dagger} P<0.05$ compared with DOX alone.

Akt activation and apoptosis. To determine whether PI3K-Akt1 or p38 MAPK is involved in CO signal transduction (14), we tracked Akt 1 and p38 MAPK phosphorylation by western blot analysis using phospho-specific antibodies (Figure 6C). Akt phosphorylation was markedly inhibited by DOX, but no significant effect of DOX was found on p38 MAPK (data not shown). Since Akt phosphorylation was restored, 2 Akt downstream target proteins, GSK3 $\beta$ and Bad, were examined by western blot analysis. DOX decreases the phosphorylation of both GSK3 $\beta$ and Bad, and this was prevented by $\mathrm{CO}$ (Figure 6C). This result strongly implicates Akt1, via Bad phosphorylation, in the protection against DOXinduced apoptosis by CO. And because Akt1 negatively regulates GSK3 $\beta$ activation, and GSK3 $\beta$ negatively regulates nuclear GATA-4 (26), GSK3 $\beta$ is implicated in the low GATA binding levels. CO increases phospho-GSK3 $\beta$ levels, decreasing GSK3 $\beta$ activation, a known factor in GATA-4 transcriptional activation (28).

HO- 1 and DOX cardiomyocyte mtDNA depletion and cytotoxicity. $\mathrm{CO}$ is known to stimulate mitochondrial heme release, which increases $\mathrm{HO}-1$ expression and endogenous CO generation (29). This effect of $\mathrm{CO}$ on $\mathrm{HO}-1$ expression was demonstrated in the mouse heart and in control exposures, showed that it was not due to $\mathrm{HH}$ (Figure 7A).

To determine the involvement of $\mathrm{HO}-1$ in the $\mathrm{CO}$ protection against $\mathrm{DOX}$, we used rat $\mathrm{H} 9 \mathrm{c} 2$ cardiomyocytes. In these cells, DOX at clinically relevant levels (30) diminishes viability in the MTT assay at a dose of $0.5 \mu \mathrm{g} / \mathrm{ml}$ at 24 hours $(46 \% \pm 5 \%$; $P<0.001)$. H9c2 cells transfected with active human HO-1 demonstrate better viability at 24 hours after this DOX dose (to $66 \% \pm 6 \%$ of control; $P<0.05)$. 
Because DOX generates ROS and selectively damages mtDNA by $\mathrm{O}_{2}$-dependent redox cycling of the quinone and semi-quinone forms (31), we tracked this damage in $\mathrm{H} 9 \mathrm{c} 2$ cells using the mtDNA/ nDNA ratio (Figure $7 \mathrm{~B}$ ). At 24 hours after DOX, the mtDNA/nDNA ratio in surviving cells had fallen to $42 \% \pm 7 \%$ that of control values $(P<0.01)$; however, cells overexpressing HO-1 displayed normal $\mathrm{mtDNA} / \mathrm{nDNA}$, and after DOX maintained their mtDNA/nDNA ratio at $79 \% \pm 9 \%$ of control $(P=0.01$ compared with DOX-treated control cells). Protection by HO- 1 requires Akt 1 , as mtDNA was not protected in DOX-treated cells preincubated with Akt inhibitor (Akt8; data not shown) or cells transfected with HO-1 and treated with Akt inhibitor (Akt8) before DOX (Figure 7B). It has been demonstrated that HO-1 activates GC and that cGMP is involved in CO-activating mitochondrial biogenesis in these cells (16). In the present studies, losses in cellular mtDNA content after DOX correlated negatively with cell viability $\left(P<0.01 ; r^{2}=-0.7\right)$.

The effector caspase- 3 was checked in these cells for activation of intrinsic apoptosis, and a marked increase in the expression of the active, cleaved form was found after DOX treatment. By 24 hours, active caspase- 3 was present in $49 \% \pm 8 \%$ of DOX-treated control cells and in only $18 \% \pm 3 \%$ of cells overexpressing HO-1 $(P<0.05)$. HO-1 overexpression by gene transfection (without DOX) had no effect on the basal active caspase- 3 level. The bottom gel in Figure 7B is a western blot demonstrating that the protective effect of $\mathrm{HO}-1$ on caspase- 3 is abolished by preincubation with Akt inhibitor (Akt8; $50 \mu \mathrm{M}) 30$ minutes before DOX. Comparable data were obtained using the $\mathrm{CO}$-producing molecule dichloromethane (data not shown). We also checked H9c2 cells for DOXinduced depletion of cellular ATP content and found that HO-1 overexpression was protective (Figure 7C).

The importance of $\mathrm{HO}-1$ in protecting mtDNA copy number was also confirmed using siRNA for HO-1. At 24 and 48 hours after siHO-1, H9c2 cells show greater than $90 \%$ loss of $\mathrm{HO}-1$ protein (Figure 7D; $50 \mu \mathrm{M}$ hemin was used as a positive control to induce HO-1). A scrambled siRNA had no effect on HO-1 protein expression (data not shown). The presence of siHO-1 did not deplete H9c2 cells of mtDNA, but the loss of HO-1 significantly worsened mtDNA depletion after DOX treatment (Figure 7E). HO-1 induction (by hemin) offered partial protection of mtDNA copy number, and this protection was independent of p38 MAPK (Figure 7E). Thus, the CO/HO system opposes DOX-induced mtDNA depletion and intrinsic apoptosis in cardiomyocytes, and Akt but not p38 activation is required in the protective mechanism.

\section{Discussion}

The lack of preventive or therapeutic options for the toxic cardiomyopathy of the cancer chemotherapeutic agent DOX reflects our current incomplete understanding of the pathogenesis. Here using combined molecular analyses and transgenic mitochondrial reporter mice, we have demonstrated that DOX suppresses cardiac mitochondrial biogenesis and that $\mathrm{CO} / \mathrm{HO}$ therapy reverses this suppression. DOX thus not only damages mitochondria but interferes with the nuclear transcriptional regulation of mitochondrial biogenesis, which is opposed by activation of the $\mathrm{CO} / \mathrm{HO}$ system. $\mathrm{CO}$ protects the heart by mitigating the loss of mtDNA, reducing oxidative stress, and preserving myofibril integrity, which attenuates cardiac apoptosis, necrosis, and fibrosis and maintains a nearly normal LV wall configuration.

The protective mechanism of $\mathrm{CO} / \mathrm{HO}$ differs uniquely from those proposed for other injuries that are reported to benefit $(32,33)$, and the administration of low dose CO for only 2 hours over a 14-day experiment establishes this protection as distinct from the transient vasodilator activity of CO. Also, to eliminate the possibility that hypoxic preconditioning played an important role in the $\mathrm{CO}$ protective mechanism, we compared the effects of an equivalent level of $\mathrm{HH}$ to that of the $\mathrm{CO}$ hypoxia. These data demonstrate that equivalent $\mathrm{HH}$ without $\mathrm{CO}$ is below the threshold for inducing HO-1 and does not prevent DOX-induced cardiac apoptosis or fibrosis.

The evidence that mitochondrial biogenesis is responsible for protection against $\mathrm{DOX}$ cardiomyopathy via $\mathrm{CO} / \mathrm{HO}$ is 3 -fold. First, the DOX-treated mouse heart fails to compensate for cardiac mtDNA depletion and mitochondrial protein oxidation, and intrinsic apoptosis is initiated prior to LV dilation and fibrosis. These findings are consistent with well-established observations that DOX causes progressive oxidative mitochondrial damage (8). Second, the nuclear transcriptional regulators for mitochondrial biogenesis that normally oppose a declining aerobic capacity do not react in the DOX heart. This inevitably compromises ATP availability and degrades contractile function. Third, DOX deregulates NO signaling and prevents HO-1 induction, root causes for the regulatory failure of mitochondrial biogenesis that introduce a vicious cycle of decreasing energy production and increasing cardiac wall stress.

This injury cycle is reversed by a subinjurious schedule of CO, a classical inhibitor of the respiratory chain with a recently discovered retrograde signal for mitochondrial biogenesis (16). The explanation that activation of mitochondrial biogenesis by $\mathrm{CO} /$ $\mathrm{HO}$ ameliorates LV chamber dilation and myocardial fibrosis fits the principle of parsimony for the integrated cardiac molecular responses, preservation of viable myocardium, and the quantitative reduction in reactive fibrosis by morphometry. It also fits the link between DOX-induced mtDNA depletion and apoptosis to the protective role of the $\mathrm{CO} / \mathrm{HO}$ system in cardiomyocytes.

Few prior studies on the development of DOX cardiomyopathy and the role of cardiomyocyte apoptosis have focused on the intrinsic pathway $(7,18,26)$. Although we found evidence of both apoptosis and necrosis, this is not uncommon with initiation of intrinsic apoptosis. Damaged mitochondria release proapoptotic factors, e.g., cytochrome $c$ and AIF, which trigger caspase-dependent and -independent apoptosis, respectively (34-36). The depletion of cardiac mtDNA is accompanied by the expected increases in cardiomyocyte caspase-3 cleavage and AIF translocation. Under physiological conditions, caspase- 3 is a pro-enzyme and AIF is sequestered inside the mitochondria $(34,35)$. After DOX exposure, apoptosis is initiated via both caspase- 3 activation and AIF nuclear translocation. Upon release by mitochondria, AIF binds to cyclophilin A to form an active cytoplasmic DNase that translocates to the nucleus and participates in chromatinolysis $(21,35,36)$. DOX-induced caspase- 3 cleavage and mitochondrial AIF release are both opposed by $\mathrm{CO}$, and this too connects mitochondrial biogenesis, which is antiapoptotic, to protection against cardiomyopathy.

Another critical aspect of DOX cardiac toxicity is loss of NO bioactivity - mainly through the eNOS transformation from a NOS to an oxygenase $(37,38)$, which decreases $\mathrm{NO}$ and increases superoxide production rates, thereby inhibiting GC activation $(39,40)$. NO regulates mitochondrial biogenesis, and eNOS gene knockout interferes with adaptive NO/cGMP signaling and genetic control of bioenergetics (14). Because CO also activates sGC and promotes mitochondrial biogenesis in part through cGMP (16), the restoration of gGMP levels in the DOX-treated heart appears to be an 
important component of this protective mechanism. Moreover, $\mathrm{CO}$ establishes mitochondrial oxidant activation of Akt1, which promotes NRF-1 phosphorylation/nuclear translocation, Tfam gene expression, and mtDNA replication (17), and Akt signaling is influenced by cGMP-dependent PI3K activation (41, 42), which confers a potent antiapoptotic effect by direct phosphorylation and inactivation of proapoptotic proteins including caspase- 3 (43). The cardiac homeostasis is thus protected by activating mitochondrial biogenesis via GC and Akt1 and subsequent downstream intrinsic antiapoptosis mechanisms.

Our data also implicate an increase in the expression of the molecular chaperone HSP-60 in protection of mitochondria by CO. HSP-60 is necessary for the importation of nuclear-encoded mitochondrial proteins by facilitation of matrix assembly through assisted protein-folding within the inner mitochondrial membrane (44-46). HSP-60 also opposes apoptosis by binding to Bax to prevent oligomer formation and mitochondrial membrane insertion (44). Thus depletion of HSP-60 by DOX allows Bax to move from cytosol to mitochondria, forming a pore and triggering apoptosome formation and caspase- 3 activation $(45,46)$.

It is also important to recognize the importance of SOD2 upregulation by CO (19) in preventing DOX toxicity. SOD2 activity certainly counteracts the participation of superoxide in mtDNA and protein oxidation, but the enzyme also supports mitochondrial biogenesis by facilitating the peroxide leak rate at Complex III (16). Moreover, CO accumulation in cells triggers mitochondrial heme release, upregulating $\mathrm{HO}-1$ and generating endogenous $\mathrm{CO}$ (29), which, as shown here, sustains mitochondrial biogenesis. On the other hand, sustained mitochondrial ROS production in heart failure is implicated in the increased rate of extracellular matrix turnover, fibrosis, and degradation of sarcomeric proteins (22).

The lack of Akt 1 phosphorylation in the evolution of cardiomyopathy implicates protein kinase deregulation in support of sarcomeric contractile-protein stability, much like the proposed basis of DOX inhibition of cardiomyocyte protein synthesis (24, 47). In contrast, Akt 1 activation by the $\mathrm{CO} / \mathrm{HO}$ system stabilizes sarcomere proteins and opposes the DOX effect. The sarcomere proteins - MHC, troponin I, and $\alpha$-actinin - are vital to the regulation of cardiac structure and contractile function $(22,47)$, e.g., coupled to intracellular calcium, and prevention of sarcomeric protein degradation exerts an overall antiatrophic effect on the myocardium. Moreover, myocardial MHC and troponin I expression are regulated by the GATA-4 transcription factor during cardiac development and differentiation (25).

GATA-4 is inactivated in DOX cardiomyopathy, and GATA-4 overexpression protects against DOX-induced apoptosis $(26,27)$, demonstrating a prosurvival function. We too have demonstrated that DOX interferes with GATA-4 DNA binding but also that CO restores cardiac GATA-4 activity, alone as well as after DOX, thereby connecting it directly to the GATA-4 antiatrophic effect. Akt 1 positively regulates GATA- 4 transactivation in cardiomyocytes via phosphorylation and inactivation of GSK3 $\beta$ (28), and here DOX reduces PGSK3 $\beta$, a negative regulator of GATA- 4 in the nucleus. CO increases GSK3 $\beta$ phosphorylation and thereby functionally inactivates GSK3 $\beta$. Moreover, in neurons, GSK3 $\beta$ phosphorylates Bax and promotes mitochondrial initiation of apoptosis (48). CO-dependent GSK3 $\beta$ inactivation would therefore oppose intrinsic apoptosis.

In summary, our findings indicate that sharp declines in mtDNA content and mitochondrial function are central to the pathogenesis of DOX cardiomyopathy in mice; however, a decisive factor in the progressive mitochondrial loss is inactivation of the transcriptional control mechanisms for mitochondrial biogenesis through the NO/cGMP and $\mathrm{CO} / \mathrm{HO}$ systems. Moreover, $\mathrm{CO} / \mathrm{HO}$ restores the nuclear regulatory program for mitochondrial biogenesis, in part by Akt1 activation, which is antiapoptotic and prosurvival, limiting the cardiomyopathy by preventing cell death, myocardial degeneration, and fibrosis. Because the antitumor properties of DOX are more closely related to its telomerase effect, we expect that efforts to protect mitochondrial biogenesis will improve the therapeutic index of this important anticancer drug. $\mathrm{CO} / \mathrm{HO}$ and/or other activators of mitochondrial biogenesis may provide a rational basis for novel cardioprotective strategies to avoid cardiomyopathy and cardiac fibrosis in at-risk patients.

\section{Methods}

Animals. We used 10- to 12-week-old male C57BL/6 mice (Jackson Laboratories) or transgenic mice that express GFP exclusively in mitochondria (mtGFP-tg mice, a gift from Hiroshi Shitara and Hiromichi Yonekawa of Tokyo Metropolitan Institute of Medical Science, Tokyo, Japan) (20). Cardiomyopathy was induced by a single i.p. injection of DOX $\mathrm{HCl}$ (SigmaAldrich) at a dose of $15 \mathrm{mg} / \mathrm{kg}$. The presence of DOX-induced cardiomyopathy was confirmed by histology and observations of cardiomyocyte disintegration compared with control mice injected with $0.9 \% \mathrm{NaCl}$.

The therapy protocols consisted of CO blended at $500 \mathrm{ppm}$ with air delivered into specially designed chambers for 1 hour to increase peak carboxyhemoglobin level to approximately $25 \%$ (IL model 482 Co-oximeter). Mice received a CO treatment 1 day before DOX or 1 treatment before and another 7 days after DOX injection. Other mice were exposed to $\mathrm{HH}$ in a high-altitude chamber (simulating 15,000 feet elevation) for 1 hour followed by a gradual return to sea level either 1 day before or 1 day before and again 7 days after DOX, following the CO treatment protocols exactly. Control groups received $2 \mathrm{CO}$ or $2 \mathrm{HH}$ treatments but no DOX injections. All mice were observed daily and sacrificed after 14 days. All procedures were approved by the Duke University Institutional Animal Care and Use Committee.

Histopathology. The gross morphology and degree of fibrosis were evaluated in hearts isolated and perfusion fixed at a constant pressure $(80 \mathrm{mmHg})$ with $10 \%$ formalin. Fixed hearts were sectioned across the ventricles (in transverse), embedded in paraffin, and sectioned at approximately $4 \mu \mathrm{m}$. Sections were stained with $\mathrm{H} \& \mathrm{E}$, and gross morphology was viewed under a low-power field $(\times 5)$. The internal circumferences of the LV and RV as well as LV wall thickness were measured. For analysis of LV fibrosis, 6 random photomicrographs were taken in viable myocardium at $\times 400$ magnification for each animal. The extent of fibrosis and myocyte diameter in these photomicrographs was quantified by a blinded observer using the ImageJ program from $\mathrm{NIH}$.

Immunofluorescence microscopy. Paraffin-embedded tissue sections $(4 \mu \mathrm{M})$ were deparaffinized in xylene and rehydrated through a series of alcohols, washed extensively in $0.1 \mathrm{M}$ PBS, blocked with $10 \%$ normal goat serum with avidin, and incubated overnight at $4{ }^{\circ} \mathrm{C}$ in primary antibody diluted in $10 \%$ NGS and biotin. Anti-laminin, anti-SOD2 (StressGen), and antiTfam primary antisera (16) were used at a dilution of 1:1000. Sections were extensively washed in PBS and incubated in secondary anti-rabbit IgG, Alexa Fluor 594. Conventional fluorescence images were obtained using a Nikon Microphot-FXA fluorescence microscope. Confocal fluorescence images were obtained using a LSCM confocal microscopy unit (Model 410; Carl Zeiss MicroImaging). Image analysis and fluorescence quantification were performed in a blinded manner using Image J from the NIH. To compare immunofluorescence stain intensities among cells 
of different sizes and shapes, single optical sections $(0.7 \mu \mathrm{m})$ were taken at a level that included the nucleus. Four uniform regions $\left(4.62 \mu \mathrm{m}^{2}\right)$ were sampled from nucleus, perinuclear cytoplasm (within $5 \mu \mathrm{m}$ of the nucleus), peripheral cytoplasm (more than $5 \mu \mathrm{m}$ from the nucleus), or processing regions of each cell. The mean pixel intensities were averaged to generate a mean value for each region of the cell. To calculate staining intensity per region, the values of each region for individual cells were averaged over 20 cells.

MtDNA copy number and encoded respiratory chain protein. mtDNA was determined by real-time PCR using SYBR green qPCR as previously described (17). Fluorescence intensities during PCR were recorded and analyzed in an ABI Prism 7000 sequence detector system (Applied Biosystems 7000 SDS software). The mtDNA encoded subunit I of COXI was also quantified by RT-PCR and normalized against nuclear-encoded 18S and GAPDH.

Western blot analysis. Proteins (total, nuclear, and mitochondrial) were separated by SDS-PAGE (19). Membranes were incubated with validated polyclonal or monoclonal antibodies against PGC-1, NRF-1, and Tfam (16); Poly, Akt, pAkt, p38, p-p38, troponin I, and $\alpha$-actinin (Santa Cruz Biotechnology Inc.); SOD2 (StressGen); GPX (Abcam); anti-HSP-60, anti-caspase-3, anti-pBad, and anti-pGSK3 $\beta$ (Cell Signaling); and anti-tubulin or $\beta$-actin (1:1,000; Sigma-Aldrich). After 5 washes in TBST, membranes were incubated in 1:10,000 HRP-conjugated goat anti-rabbit or anti-mouse IgG (Amersham Biosciences). Blots were developed with ECL, and proteins were quantified on digitized images from the mid-dynamic range and expressed relative to tubulin or $\beta$-actin.

Protein carbonylation. Cardiac mitochondrial protein $(20 \mu \mathrm{g})$ was derivatized with dinitrophenylhydrazine and slot-blotted onto Hybond-polyvinylidene difluoride membranes. Carbonyls were detected with rabbit antidinitrophenol (DNP) (1:250; Intergen) and HRP-conjugated anti-rabbit $\operatorname{IgG}(1: 1000)$ using the ECL system (Amersham Biosciences). Band densities were quantified by densitometry using Quantity One Software (BioRad). Optical density results were expressed as percent of control values.

Myocardial cGMP levels. Frozen myocardial tissue samples in liquid nitrogen were ground to a fine powder in a stainless steel mortar. After evaporating the liquid nitrogen, the frozen tissue was weighed and homogenized in 10 volumes $0.1 \mathrm{M} \mathrm{HCl}$ and centrifuged at $20,000 \mathrm{~g}$ at room temperature, and the supernatant was collected for quantitative immunoassay of cGMP level by ELISA (CG-200; Sigma-Aldrich). The assay aliquots were added to 96-well plates precoated with anti-rabbit Ab cGMP-peroxidase conjugate, washed, and peroxidase substrate added. The reaction was terminated with $\mathrm{H}_{2} \mathrm{SO}_{4}$, and $\mathrm{OD}_{450}$ was read against standards.

MHC isoform analysis. LV tissues were homogenized in $250 \mathrm{mM}$ sucrose, $100 \mathrm{mM} \mathrm{KCl}, 5 \mathrm{mM}$ EDTA, and $20 \mathrm{mM}$ Tris base, $\mathrm{pH}$ 6.8, sonicated, and centrifuged for 10 minutes at $1,000 \mathrm{~g}\left(4^{\circ} \mathrm{C}\right)$. The pellet was suspended in buffer $(175 \mathrm{mM} \mathrm{KCl}, 0.5 \%$ Triton X-100, 2 mM EDTA, $20 \mathrm{mM}$ Tris base, $\left.\mathrm{pH} 6.8,4^{\circ} \mathrm{C}\right)$ and centrifuged for 10 minutes at $1,000 \mathrm{~g}\left(4^{\circ} \mathrm{C}\right)$. The pellet was suspended in buffer again and centrifuged for 10 minutes at $1,000 \mathrm{~g}\left(4^{\circ} \mathrm{C}\right)$ and then resuspended in a final buffer $(150 \mathrm{mM} \mathrm{KCl}$ and $20 \mathrm{mM}$ Tris base, $\mathrm{pH}$ 7.0). Total protein was determined and concentration adjusted to $6 \mathrm{mg} / \mathrm{ml}$ in the final buffer. Samples were diluted to $0.125 \mathrm{mg} / \mathrm{ml}$ with $2 \times$ Laemmli sample buffer with $1 \% \beta$-mercaptoethanol and $0.2 \%$ bromophenol blue and boiled for 2 minutes, cooled on ice, and loaded onto polyacrylamide gels. MHC isoforms were separated via SDS-PAGE using stacking gels consisting of $4 \%$ acrylamide-bis and separating gels consisting of $8 \%$ acrylamide-bis. Samples ( $2 \mu \mathrm{g}$ protein) were loaded onto gels and electrophoresed at $100 \mathrm{~V}$ followed by silver staining (Bio-Rad Silver stain plus). Gels were scanned using Quantity One Software (Bio-Rad).

GATA DNA binding activity. GATA-4 DNA binding activity in nuclear protein extracts was analyzed by EMSA using double-stranded consen- sus GATA-4-specific oligonucleotides (sense, 5'-TCGCTGGACTGATAACTTTAAAAG-3') (17).

Human HO-1 cDNA plasmid construction, HO-1 transfection, and HO-1 silencing. A hHO-1 construct was prepared consisting of pcDNA 3.1/V5His-hHO-1 containing the entire protein-coding region (866 bp) of the human HO-1 gene. The HO-1 plasmid construct assembly was enabled using forward ( $5^{\prime}$-TTCATACAAGCTTATGGAGCGTCCGCAACC-3') and reverse primers (5'-TCAATGGATCCTCACATGGCATAAAGCCCT- $\left.3^{\prime}\right)$ and amplified by $p f u$ DNA polymerase-catalyzed PCR and adenine overhangs added to the PCR product with Taq DNA polymerase. After purification of the PCR products, the hHO-1 fragment was subcloned into pcDNA 3.1/ V5-His TOPO TA vector (Invitrogen). Identical plasmids minus hHO-1 cDNA were used for control transfection. Correct sense orientation and hHO-1 construct sequence were confirmed by sequencing both strands.

Cell studies. H9c2 cells (embryonic rat cardiomyocytes; ATCC) were maintained in DMEM (Invitrogen) supplemented with 10\% fetal bovine serum (Invitrogen) and $2 \mathrm{mmol} / \mathrm{l} \mathrm{L}$-glutamine. Cells were plated on 35- or 100-mm dishes in DMEM with 10\% FCS and used when 70\%-90\% confluent. DOX was added to complete medium and incubated at $37^{\circ} \mathrm{C}$ for various intervals at a concentration $(0.5 \mu \mathrm{g} / \mathrm{ml})$ similar to plasma concentrations encountered in clinical use (30).

The role of HO- 1 on ameliorating DOX-induced cell damage was addressed in cardiomyocytes using $\mathrm{HO}-1$ transfection, $\mathrm{HO}-1$ induction with hemin, or HO-1 induction with siRNA for HO-1. Cells $\left(1 \times 10^{6}\right)$ were transfected transiently with $4 \mu \mathrm{g}$ of plasmid DNA-Lipofectamine 2000 complex according to the manufacturer's instructions (Invitrogen). This transfection dose was shown to elicit 3- to 4-fold increases in total HO enzyme activity relative to sham-transfected cells. Following incubation for 6 hours at $37^{\circ} \mathrm{C}$, the transfection mixture was replaced with $10 \mathrm{ml}$ of complete media without antibiotics. A transfection efficiency of approximately $60 \%$ was determined by GFP expression in $\mathrm{H} 9 \mathrm{c} 2$ cells cotransfected with hHO-1 cDNA plasmid and pEGFP.C1 vector (Clontech Laboratories). After 48 hours, HO1 and empty-plasmid transfected cells were treated with DOX $(0.5 \mu \mathrm{g} / \mathrm{ml}$; Sigma-Aldrich). A similar protocol was followed for HO-1 induction with hemin $(50 \mu \mathrm{M})$, except that DOX was added after 6 hours.

In separate experiments, $\mathrm{H} 9 \mathrm{c} 2$ cells were transfected transiently with $100 \mathrm{nM}$ HO-1 siRNA (Santa Cruz Biotechnology Inc.) mixed with FuGENE-HD (Roche) as previously reported (16). To check for the involvement of p38 MAPK, transfected cells were preincubated with SB203580 (10 $\mu \mathrm{M})$ for 60 minutes, then treated for an additional 6 hours with hemin before DOX. Cell viability was tracked by MTT assay, and cells were also collected for mitochondrial and nuclear DNA extraction, protein analysis, and ATP assays. ATP was measured in cell lysates using a standard enzymatic assay based on hexokinase and glucose-6-phosphate dehydrogenase. The effect of DOX and the interventions above on mtDNA copy number was assessed by real-time PCR.

\section{Acknowledgments}

The authors thank John Patterson and Lynn Tatro for technical assistance. This work was supported by NIH grant PO1 42-444-15 (C.A. Piantadosi).

Received for publication June 12, 2007, and accepted in revised form September 26, 2007.

Address correspondence to: Claude A. Piantadosi, Department of Medicine, 0590 Clinical Research Building II, South Hospital, Box 3315, 200 Trent Drive, Durham, North Carolina 27710, USA. Phone: (919) 684-6143; Fax: (919) 684-6002; E-mail: piant001@ mc.duke.edu. 
1. Steinherz, L.J., Steinherz, P.G., and Tan, C. 1995. Cardiac failure and dysrhythmias 6-19 years after anthracycline therapy: a series of 15 patients. Med. Pediatr. Oncol. 24:352-361.

2. Swain, S.M., Whaley, F.S., and Ewer, M.S. 2003. Congestive heart failure in patients treated with doxorubicin: a retrospective analysis of three trials. Cancer. 97:2869-2879.

3. DeGraff, W., Hahn, S.M., Mitchell, J.B., and Krishna, M.C. 1994. Free radical modes of cytotoxicity of adriamycin and streptonigrin. Biochem. Pharmacol. 48:1427-1435.

4. Olson, R.D., and Mushlin, P.S. 1990. Doxorubicin cardiotoxicity: analysis of prevailing hypotheses. FASEB J. 4:3076-3086.

5. Narula, J., et al. 1996. Apoptosis in myocytes in endstage heart failure. N. Engl. J. Med. 335:1182-1189.

6. Singal, P.K., and Iliskovic, N. 1998. Doxorubicin-induced cardiomyopathy. N. Engl. J. Med. 339:900-905.

7. Sawyer, D.B., Fukazawa, R., Arstall, M.A., and Kelly, R.A. 1999. Daunorubicin-induced apoptosis in rat cardiac myocytes is inhibited by dexrazoxane. Circ. Res. 84:257-265.

8. Zhou, S., Starkov, A., Froberg, M.K., Leino, R.L., and Wallace, K.B. 2001. Cumulative and irreversible cardiac mitochondrial dysfunction induced by doxorubicin. Cancer Res. 61:771-777.

9. Wallace, K.B. 2003. Doxorubicin-induced cardiac mitochondrionopathy. Pharmacol. Toxicol. 93:105-115.

10. Nakai, A., et al. 2007. The role of autophagy in cardiomyocytes in the basal state and in response to hemodynamic stress. Nat. Med. 13:619-624.

11. McLeod, C.J., Pagel, I., and Sack, M.N. 2005. The mitochondrial biogenesis regulatory program in cardiac adaptation to ischemia--a putative target for therapeutic intervention. Trends Cardiovasc. Med. 15:118-123.

12. Maines, M.D. 1988. Heme oxygenase: function, multiplicity, regulatory mechanisms, and clinical applications. FASEB J. 2:2557-2568.

13. Furchgott, R.F., and Jothianandan, D. 1991. Endothelium-dependent and -independent vasodilation involving cyclic GMP: relaxation induced by nitric oxide, carbon monoxide and light. Blood Vessels. 28:52-61.

14. Nisoli, E., et al. 2003. Mitochondrial biogenesis in mammals: the role of endogenous nitric oxide. Science. 299:896-899.

15. Nisoli, E., et al. 2004. Mitochondrial biogenesis by NO yields functionally active mitochondria in mammals. Proc. Natl. Acad. Sci. U. S. A. 101:16507-16512.

16. Suliman, H.B., Carraway, M.S., Tatro, L.G., and Piantadosi, C.A. 2007. A new activating role for $\mathrm{CO}$ in cardiac mitochondrial biogenesis. J. Cell Sci. 120:299-308.

17. Piantadosi, C.A., and Suliman, H.B. 2006. Mitochondrial transcription factor A induction by redox activation of nuclear respiratory factor 1 .
J. Biol. Chem. 281:324-333.

18. Childs, A.C., Phaneuf, S.L., Dirks, A.J., Phillips, T., and Leeuwenburgh, C. 2002. Doxorubicin treatment in vivo causes cytochrome $\mathrm{C}$ release and cardiomyocyte apoptosis, as well as increased mitochondrial efficiency, superoxide dismutase activity, and Bcl-2:Bax ratio. Cancer Res. 62:4592-4598.

19. Piantadosi, C.A., Carraway, M.S., and Suliman, H.B. 2006. Carbon monoxide, oxidative stress, and mitochondrial permeability pore transition. Free Radic. Biol. Med. 40:1332-1339.

20. Shitara, H., et al. 2001. Non-invasive visualization of sperm mitochondria behavior in transgenic mice with introduced green fluorescent protein (GFP). FEBS Lett. 500:7-11.

21. Wang, H., et al. 2004. Apoptosis-inducing factor substitutes for caspase executioners in NMDAtriggered excitotoxic neuronal death. J. Neurosci. 24:10963-10973.

22. Murdoch, C.E., Zhang, M., Cave, A.C., and Shah, A.M. 2006. NADPH oxidase-dependent redox signalling in cardiac hypertrophy, remodelling and failure. Cardiovasc. Res. 71:208-215.

23. Cole, M.P., et al. 2006. The protective roles of nitric oxide and superoxide dismutase in adriamycininduced cardiotoxicity. Cardiovasc. Res. 69:186-197.

24. Molkentin, J.D., Kalvakolanu, D.V., and Markham, B.E. 1994. Transcription factor GATA-4 regulates cardiac muscle-specific expression of the alpha-myosin heavy-chain gene. Mol. Cell. Biol. 14:4947-4957.

25. Murphy, A.M., Thompson, W.R., Peng, L.F., and Jones, L., 2nd. 1997. Regulation of the rat cardiac troponin I gene by the transcription factor GATA-4. Biochem. J. 322:393-401.

26. Kim, Y., et al. 2003. Anthracycline-induced suppression of GATA-4 transcription factor: implication in the regulation of cardiac myocyte apoptosis. Mol. Pharmacol. 63:368-377.

27. Aries, A., Paradis, P., Lefebvre, C., Schwartz, R.J., and Nemer, M. 2004. Essential role of GATA-4 in cell survival and drug-induced cardiotoxicity. Proc. Natl. Acad. Sci. U. S. A. 101:6975-6980.

28. Morisco, C., et al. 2001. Glycogen synthase kinase 3 beta regulates GATA4 in cardiac myocytes. J. Biol. Chem. 276:28586-28597.

29. Cronje, F.J., et al. 2004. Carbon monoxide actuates $\mathrm{O}(2)$-limited heme degradation in the rat brain. Free Radic. Biol. Med. 37:1802-1812.

30. Gianni, L., et al. 1997. Human pharmacokinetic characterization and in vitro study of the interaction between doxorubicin and paclitaxel in patients with breast cancer. J. Clin. Oncol. 15:1906-1915.

31. Singh, M., Atwal, H., and Micetich, R. 1998. Transferrin directed delivery of adriamycin to human cells. Anticancer Res. 18:1423-1427.

32. Wagener, F.A., et al. 2003. Different faces of the heme-heme oxygenase system in inflammation. Pharmacol. Rev. 55:551-571.

33. Bak, I., Varadi, J., Nagy, N., Vecsernyes, M., and Tosaki, A. 2005. The role of exogenous carbon monoxide in the recovery of post-ischemic cardiac function in buffer perfused isolated rat hearts. Cell. Mol. Biol. (Noisy-le-grand) 51:453-459.

34. Lorenzo, H.K., Susin, S.A., Penninger, J., and Kroemer, G. 1999. Apoptosis inducing factor (AIF): a phylogenetically old, caspase-independent effector of cell death. Cell Death Differ. 6:516-524.

35. Ye, H., et al. 2002. DNA binding is required for the apoptogenic action of apoptosis inducing factor. Nat. Struct. Biol. 9:680-684.

36. Yu, S.W., et al. 2002. Mediation of poly(ADP-ribose) polymerase-1-dependent cell death by apoptosisinducing factor. Science. 297:259-263.

37. Duquaine, D., et al. 2003. Rapid-onset endothelial dysfunction with adriamycin: evidence for a dysfunctional nitric oxide synthase. Vasc. Med. 8:101-107.

38. Vasquez-Vivar, J., et al. 1997. Endothelial nitric oxide synthase-dependent superoxide generation from adriamycin. Biochemistry. 36:11293-11297.

39. Unverferth, D.V., et al. 1981. The effect of firstdose doxorubicin on the cyclic nucleotide levels of the human myocardium. Toxicol. Appl. Pharmacol. 60:151-154.

40. Lehotay, D.C., Levey, B.A., and Levey, G.S. 1983. Inhibition of cardiac guanylate cyclase by doxorubicin and some of its analogs. Biomed. Pharmacother. 37:312-316.

41. Ha, K.S., et al. 2003. Nitric oxide prevents 6-hydroxydopamine-induced apoptosis in PC12 cells through cGMP-dependent PI3 kinase/Akt activation. FASEB J. 17:1036-1047.

42. Das, A., Smolenski, A., Lohmann, S.M., and Kukreja, R.C. 2006. Cyclic GMP-dependent protein kinase Ialpha attenuates necrosis and apoptosis following ischemia/reoxygenation in adult cardiomyocyte. J. Biol. Chem. 281:38644-38652.

43. Datta, S.R., Brunet, A., and Greenberg, M.E. 1999. Cellular survival: a play in three Akts. Genes Dev. 13:2905-2927.

44. Kirchhoff, S.R., Gupta, S., and Knowlton, A.A. 2002. Cytosolic heat shock protein 60, apoptosis, and myocardial injury. Circulation. 105:2899-2904.

45. Jurgensmeier, J.M., et al. 1998. Bax directly induces release of cytochrome $\mathrm{c}$ from isolated mitochondria. Proc. Natl. Acad. Sci. U. S. A. 95:4997-5002.

46. Bossy-Wetzel, E., Newmeyer, D.D., and Green, D.R. 1998. Mitochondrial cytochrome c release in apoptosis occurs upstream of DEVD-specific caspase activation and independently of mitochondrial transmembrane depolarization. EMBOJ. 17:37-49.

47. Ito, H., et al. 1990. Doxorubicin selectively inhibits muscle gene expression in cardiac muscle cells in vivo and in vitro. Proc. Natl. Acad. Sci. U. S. A. 87:4275-4279.

48. Linseman, D.A., et al. 2004. Glycogen synthase kinase3 beta phosphorylates Bax and promotes its mitochondrial localization during neuronal apoptosis. J. Neurosci. 24:9993-10002. 